FORMATION Formation emploi

Revue française de sciences sociales

137 | Janvier-Mars 2017

La formation continue en contexte : l'entreprise au cœur des enjeux

\title{
Les pratiques managériales pour réduire les inégalités professionnelles de genre ? L'exemple de la promotion et de la formation en entreprise
}

Can managerial practices reduce gender inequalities in the workplace? The case of promotion and training

Managementpraktiken zur Reduzierung der Geschlechterungleichheiten im Beruf? Untersuchung am Beispiel der innerbetrieblichen Aufstiegschancen und Ausbildung

¿Prácticas gerenciales para reducir las desigualdades profesionales de género?

El ejemplo de la promoción y la formación en empresa

Ekaterina Melnik-Olive et Hélène Couprie

\section{(2) OpenEdition}

\section{Journals}

Édition électronique

URL : http://journals.openedition.org/formationemploi/5013

DOI : 10.4000/formationemploi.5013

ISSN : 2107-0946

Éditeur

La Documentation française

Édition imprimée

Date de publication : 30 avril 2017

Pagination : 97-117

ISSN : 0759-6340

Référence électronique

Ekaterina Melnik-Olive et Hélène Couprie, «Les pratiques managériales pour réduire les inégalités professionnelles de genre ? L'exemple de la promotion et de la formation en entreprise ", Formation emploi [En ligne], 137 | Janvier-Mars 2017, mis en ligne le 30 avril 2019, consulté le 21 décembre 2020. URL : http://journals.openedition.org/formationemploi/5013 ; DOI : https://doi.org/10.4000/ formationemploi.5013 


\title{
Les pratiques managériales pour réduire les inégalités professionnelles de genre? L'exemple de la promotion et de la formation en entreprise
}

\author{
Ekaterina Melnik-Olive \\ Chargée d'études au Céreq, membre associée à Aix-Marseille Univ., CNRS, LEST, \\ Aix-en-Provence, France
}

HÉLĖne COUPRIE Maîtresse de conférences en sciences économiques à l'université de Cergy-Pontoise, laboratoire THEMA - Théorie économique, modélisation et applications (UMR 8184)

Résumé

Les pratiques managériales pour réduire les inégalités de genre ? L'exemple de la promotion et de la formation en entreprise

L'article s'intéresse au rôle des pratiques de gestion des ressources humaines dans la réduction des inégalités professionnelles de genre, abordées sous l'angle de l'accès à la promotion et à la formation en entreprise. Nous distinguons la formation en situation de travail de celle de type cours ou stage, en supposant qu'il existe une différence dans les mécanismes de sélection. Les données quantitatives mobilisées sont issues des enquêtes couplées Entreprise-Salarié (Difes 2) produites par le Céreq. Les principaux résultats montrent l'impact positif d'une politique structurée de formation et de développement des compétences sur les chances de promotion des femmes.

Mots clés : pratique de GRH, promotion, accès a la FPC, genre, FPC en entreprise, division sexuelle du travail

Abstract

Can managerial practices reduce gender inequalities in the workplace? The case of promotion and training

The paper addresses the role of HR practices in reducing gender inequalities in workplace. The gender inequalities are assessed through the access to promotion and two types of continuing vocational training in enterprise ("on-the job" and "general"). The data used are from the survey DIFES 2 combining responses of workers' and employers' conducted by the Céreq. Our results show a strong positive impact of a structured HR policy on the chances of promotion for women. Without specifying any causality, the 
study reveals a stronger link between promotion and corporate training for women than for men.

Keywords: HR management practice, promotion, access to CVT, gender, CVT in companies, gender based division of labour

Journal of Economic Literature: J 24 ; M 51 ; J 71

Traduction : auteures

Le monde de l'entreprise constitue la cible privilégiée des politiques de promotion de l'égalité professionnelle entre hommes et femmes. Pourtant, les travaux consacrés au rôle des pratiques organisationnelles sur les inégalités de genre dans le monde professionnel restent relativement rares au regard de l'ampleur de la littérature analysant les déterminants familiaux et institutionnels. Cet article étudie le rôle des pratiques de gestion des ressources humaines $(\mathrm{GRH})$ dans la réduction des inégalités professionnelles abordées sous l'angle de l'accès à la promotion et à la formation en entreprise.

Les inégalités professionnelles sont aujourd'hui un phénomène bien connu. En France, le salaire moyen horaire net des femmes est inférieur de $18 \%$ à celui des hommes. Plus de la moitié de cet écart reste inexpliquée par les caractéristiques individuelles et celles relatives à l'emploi (DARES, 2015). La ségrégation professionnelle (une surreprésentation féminine dans les emplois moins propices à la progression de carrière) et les effets des représentations sociales sur les carrières féminines sont les facteurs fréquemment avancés pour expliquer les différences de salaire. En France, à caractéristiques égales, les femmes ont moins de chances que les hommes d'accéder à des postes proposant des salaires élevés (Gobillon et al., 2015). Elles connaissent plus souvent des parcours professionnels discontinus, des temps partiels dus aux obligations familiales, ce qui a des conséquences sur leurs carrières et leurs revenus (Meurs et al., 2011). Les carrières féminines sont freinées par des interruptions, mais aussi par des obstacles " invisibles ", créés par des représentations sociales stéréotypées, la surreprésentation masculine sur les postes clés et dans les réseaux professionnels.

Pourtant, en France, l'égalité professionnelle fait l'objet d'un imposant dispositif juridique : plusieurs lois ont été promulguées entre 1972 et 2014. D’abord relatifs aux inégalités salariales, les textes ont progressivement élargi leur domaine d'action à tout le champ professionnel. Actuellement, la loi impose une démarche de suivi des inégalités professionnelles de genre aux entreprises de plus de 50 salariés. L'accent est mis sur la négociation collective et la définition des objectifs à atteindre dans différents domaines, dont la formation et la promotion.

Cependant, ce type de dispositif rencontre des limites qui semblent témoigner d'un manque d'appropriation des outils de suivi et de négociation par certaines entreprises (Brunet et Dumas, 2012 ; Laufer et Silvera, 2006). Par exemple, la couverture des 
accords d'entreprise abordant l'égalité professionnelle reste relativement faible (12\% en 2013 et $14 \%$ en 2014, selon la DARES, 2015). Elle varie en outre selon la taille de l'entreprise (seulement 29,5\% des entreprises de 50 à 300 salariés couvertes contre 63,5\% des entreprises de 300 à 1000 salariés et 75,4\% des entreprises de plus de 1000 salariés). Les rapports de situation comparée requis ne sont pas toujours produits; de même, le contenu des accords d'entreprise n'est pas toujours relié aux résultats des diagnostics (Brunet et Dumas, 2012 ; Becker et Derrien-Cortès, 2014).

Dans une optique d'affirmation de la responsabilité sociale, certaines entreprises adoptent des démarches volontaristes visant à promouvoir l'égalité professionnelle entre hommes et femmes (Landrieux-Kartochian, 2007 ; Klarsfeld et al., 2012).

Cet article constitue une synthèse d'un document de travail récent (Couprie et Melnik, 2016). Il explore un troisième levier de réduction des inégalités professionnelles. Il se focalise sur la façon dont la gestion de la formation et des compétences en entreprise s'articule avec les différences d'accès à la promotion et à la formation entre hommes et femmes.

Tandis que les inégalités salariales occupent une place centrale dans les préoccupations des acteurs, les différences d'évolution de carrière constituent un déterminant important de ces inégalités (Charbeau et de Larquier, 2010). Or, en tant qu'aspects du développement de carrière, la formation et la promotion font partie d'un même processus influencé par un ensemble de pratiques organisationnelles (Solomon et al., 1986). Sans chercher à établir une relation causale entre la formation et la promotion, nous les étudions comme deux processus reliés au sein desquels les mécanismes de sélection (ou d'auto-sélection) des salariés peuvent jouer un rôle important. Nous étudions les inégalités d'accès à la promotion et à la formation en entreprise à partir des données d'enquêtes couplées issues du "Dispositif d'Information sur la Formation EmployeurSalarié " (Difes 2) (voir encadré 1). Ces données regroupent les réponses des salariés et des employeurs. Cela permet d'analyser l'accès à la promotion et à la formation des salariés en lien avec les pratiques RH mises en place par les entreprises.

Nous identifions les pratiques RH susceptibles d'agir sur la réduction des inégalités professionnelles et élaborons un indicateur composite permettant d'approcher un ensemble de pratiques cohérentes entre elles. Après avoir constaté les écarts dans les taux d'accès à la promotion, défavorables aux femmes, nous étudions les effets de l'indicateur RH sur les chances de promotion et de formation selon le genre, en admettant un lien différencié entre la promotion et la formation selon les types de formation.

Nos résultats révèlent que les pratiques $\mathrm{RH}$ peuvent avoir des effets sur la réduction des écarts d'accès à la promotion selon le genre. Pour conclure, nous amorçons une discussion autour des enjeux de la gestion des ressources humaines au sein des organisations. 


\section{Encadré 1 : Les données d'enquêtes couplées employeur-salarié Difes 2}

Le « Dispositif d'Information sur la Formation Employeur-Salarié » (Difes 2) est un dispositif d'enquêtes couplées portant sur environ 6000 salariés et 1800 entreprises d'au moins 10 salariés. Ce dispositif, mis en place par le Céreq, contient deux volets : Entreprise et Salarié.

Le premier présente une interrogation des entreprises sélectionnées à partir d'un échantillon des 5360 entreprises ayant répondu à l'enquête européenne CVTS (Continuing Vocational Training Survey). Les entreprises ont été interrogées en 2011 sur leurs pratiques dans le domaine de la formation continue et de la gestion des compétences durant l'année 2010.

Le volet « Salarié » interroge, en 2012, les salariés des entreprises échantillonnées sur les changements vécus, ainsi que sur les formations suivies en 2010. Les salariés interrogés sont représentatifs de 13,5 millions de personnes présentes en 2010 dans les entreprises de 10 salariés et plus (hors santé, agriculture, éducation).

Nos résultats ne portent pas sur le secteur public, hors champ et régi par des règles de promotion spécifiques. Les plus petites entreprises, pour lesquelles l'accès à la promotion nécessiterait des modes d'observations spécifiques, sont également hors champ. Le mode d'interrogation rétrospectif peut générer un biais de mémoire qui ne devrait toutefois pas affecter nos résultats dans une direction spécifique.

\section{Favoriser le dialogue, la transparence et la réflexion en termes de formation et de carrières}

La ségrégation professionnelle en fonction du genre, les stéréotypes et la culture organisationnelle font partie des sources des inégalités professionnelles. Nous nous intéressons à la façon dont les pratiques $\mathrm{RH}$, au sein des organisations, peuvent agir sur ces aspects et élaborons un indicateur composite regroupant les pratiques $\mathrm{RH}$ a priori favorables à la réduction des inégalités.

\subsection{La ségrégation, les stéréotypes et la culture organisationnelle comme sources des inégalités}

L'analyse de la littérature fait ressortir deux principales catégories de sources d'inégalités professionnelles hommes-femmes : "structurales ", relevant plutôt de la ségrégation des métiers selon le genre ; et "culturelles ", liées à la culture managériale et à l'existence des stéréotypes.

La première catégorie d'explications porte sur la ségrégation des métiers en fonction du genre, ou effet de structure : généralement, les femmes sont concentrées dans les métiers moins propices à la progression de carrière (par exemple les fonctions support). Différents facteurs permettent de pallier les barrières visibles et invisibles créées par la ségrégation genrée des métiers : une attention particulière accordée à l'accès des 
femmes aux postes de management, une organisation du travail favorisant les interactions entre les différents métiers, ainsi que les actions de mentorat (Kalev et al., 2006 ; Cohen et Huffman, 2007). Par ailleurs, des pratiques telles que la rotation sur différents postes permettent au salarié d'élargir son cercle relationnel au travail et d'accéder à de nouvelles expériences ; de même, la mise en place d'équipes autonomes peut favoriser les relations entre les groupes professionnels. Ainsi, les interactions au travail entre les différentes catégories de personnel contribuent à estomper les frontières entre les emplois. Elles permettent également aux catégories de salariés les moins qualifiées de progresser en entreprise (Kalev, 2009).

La deuxième catégorie d'explications attribue la cause des inégalités à l'existence de stéréotypes et représentations liés au genre ou biais sociocognitifs (Ridgeway et Corell, 2004 ; Dencker, 2008). La notion de stéréotype renvoie à un mécanisme cognitif conduisant à attribuer des caractéristiques à des individus selon leur appartenance à une catégorie ou un groupe ${ }^{1}$. La différenciation sociale des rôles et des responsabilités selon le genre est omniprésente et traverse de multiples sphères, dont le monde du travail (Reskin et Bielby, 2005). Ainsi, la " culture masculine " apparaît comme l'un des déterminants des écarts de promotion entre hommes et femmes : dans certaines entreprises, les femmes cadres progressant hiérarchiquement auraient tendance à adopter un comportement "que la culture prévalente d'une société considère comme spécifique au sexe masculin" (Belghiti-Mahut, 2004, p. 150). Des compétences techniques, acquises en formation, peuvent s'avérer moins soumises aux biais que celles associées à des modèles de comportement, notamment les facultés managériales (Dickens, 1998).

Les contraintes qui pèsent sur les femmes contribuent à entretenir et généraliser certains stéréotypes. Les femmes sont plus souvent amenées à suivre leur conjoint en cas de promotion avec mobilité géographique, mais le contraire est rare (Pochic, 2005). Le risque est donc d'attribuer faussement le refus de mobilité au manque d'ambition ou de compétitivité. On observe aussi, dans certaines entreprises, un amalgame entre l'engagement personnel, la performance et la disponibilité présentielle, ancré dans la culture managériale (Pochic, 2005 ; Charbeau et de Larquier, 2010 ; Ponchut et Barth, 2012). La prégnance des normes de "présentéisme " (les temps pleins, la présence tardive au travail) ralentit la progression de carrière des femmes : "le calendrier de la vie professionnelle reste dominé par le modèle masculin (...) Riche en promotions et en formations entre 25 et 40 ans, il suppose que l'individu (...) se montre disponible au cours de ces précieuses années " (Fournier, 2001, p. 4).

1. Pour désigner le comportement basé sur les croyances généralisées, les économistes parlent de discrimination statistique. 


\subsection{Les pratiques RH qui agissent sur les inégalités de genre}

L'approche sociocognitive souligne l'impact des représentations sociales sur les pratiques des acteurs. Les travaux se référant à la théorie de la bureaucratie Weberienne postulent que les pratiques $\mathrm{RH}$ formelles permettraient de réduire les biais en rendant les décisions des managers plus objectives. La définition d'un système de classification salariale et la mise en place d'une description des postes constituent autant d'outils formels permettant, en principe, de rendre les décisions des managers plus objectives et d'éviter les discriminations. La diffusion de comptes rendus écrits améliorerait la transparence et fournirait une base pour les recours éventuels. Les grilles d'évaluation permettraient de réduire l'impact des jugements personnels sur l'évaluation des salariés.

Il n'existe toutefois pas de consensus quant aux effets empiriques des pratiques formelles sur les inégalités professionnelles (Huffman, 1995 ; Kalev et al. 2006 ; Dencker, 2008 ; Kalev, 2009 ; Dobbin et al., 2015).

Au contraire, de Larquier et Marchal (2014) soulignent que certaines pratiques formelles peuvent limiter la diversité d'embauche dans les organisations. Pour Bielby (2000), baser l'évaluation des salariés sur certains critères formels peut défavoriser les femmes. Par exemple, introduire une condition d'ancienneté pour promouvoir peut avoir des effets indésirables si l'entrée dans un métier est historiquement limitée pour les femmes. De même, la promotion basée sur le critère du diplôme ne permet pas de corriger les éventuels effets de la ségrégation éducative (Couppié et Epiphane, 2006). L'exigence d'un service ininterrompu pour les promotions peut également défavoriser les femmes.

Les pratiques formelles ne favorisent donc pas toujours l'égalité professionnelle entre les différentes catégories de salariés; certaines peuvent même les introduire de manière non intentionnelle. Ainsi, Dobbin et al. (2015) distinguent plusieurs types de pratiques RH formelles. L'existence d'un système permettant de suivre les objectifs en matière d'égalité professionnelle, la plus grande transparence ainsi que les pratiques engageant les managers paraissent favorables à l'égalité professionnelle en entreprise. En revanche, les méthodes formalisées de prise de décision, perçues parfois comme contraignantes (par exemple l'usage de grilles formalisées d'évaluation), peuvent être contre-productives, en raison notamment du manque d'adhésion des managers.

Nous nous inspirons des travaux dédiés aux effets des pratiques formelles sur les inégalités professionnelles, tout en adoptant une vision nuancée des effets potentiels des différentes pratiques (Bielby, 2000 ; Dobbin et al., 2015). Les pratiques décrites dans la littérature peuvent être classées en trois groupes ni exclusifs, ni exhaustifs : celles favorisant la transparence et la circulation de l'information; celles qui structurent la gestion des compétences et de la formation en entreprise; et enfin celles relatives au dialogue avec les salariés et leurs représentants. 
Le premier groupe de pratiques se référe à la transparence et à la circulation de l'information. Il est censé correspondre à une politique RH plus inclusive qui crée des conditions permettant de rendre la formation continue en entreprise accessible au plus grand nombre. Par exemple, une information plus transparente permettrait de réduire l'influence de l'appartenance à des réseaux dans l'entreprise, potentiellement favorable aux catégories avantagées. Ces pratiques contribueraient à réduire des inégalités, notamment dans l'accès à la formation ; en effet, leur apport le plus important concerne les salariés moins qualifiés, pour lesquels les canaux d'information "privés » sont moins accessibles (Sigot et Véro, 2009).

Le deuxième groupe relève de pratiques permettant un suivi ainsi qu'une réflexion structurée sur la gestion des compétences et de la formation en entreprise. Ces pratiques sont censées inscrire la formation et la promotion des salariés dans la stratégie de l'entreprise. L'objectivité des critères liés aux compétences permettrait de réduire la place des représentations dans les décisions. De plus, les dispositifs d'analyse et de suivi contribueraient à une meilleure connaissance des écarts dans l'accès à la formation et à la promotion dans les entreprises.

Le troisième groupe est relatif aux dispositifs de dialogue avec les salariés et leurs représentants. Un cadre propice au dialogue permettrait au plus grand nombre de salariés de faire connaître la vision qu'ils ont de leur carrière. Dans un sens, ce dialogue peut contribuer à réduire l'impact des représentations stéréotypées des managers quant aux attentes et aux capacités des salariés. Il pourrait également fournir des éléments qualitatifs sur les obstacles à la promotion et à la formation, invisibles dans les analyses chiffrées (Charbeau et de Larquier, 2010).

Ces trois groupes de pratiques pourraient permettre d'améliorer les chances de promotion des femmes par rapport aux hommes via leurs effets sur la sélection des salariés dans la promotion et la formation en entreprise. Afin de vérifier cette hypothèse, nous élaborons un indicateur composite des pratiques $\mathrm{RH}$, présenté dans la partie suivante (encadré 2). Il s'agit d'étudier ses effets sur les écarts de promotion et de formation en entreprise entre les hommes et les femmes.

\subsection{Approcher une logique organisationnelle par un indicateur composite}

L'indicateur des pratiques RH a pour but d'approcher une certaine logique organisationnelle de l'entreprise, caractérisée par la transparence de l'information, une réflexion structurée autour de la gestion des compétences et par le dialogue avec les salariés et leurs représentants.

Lidée sous-jacente est que la formation et la promotion relèvent d'un processus de sélection en entreprise pouvant être défavorable aux femmes en raison, notamment, des biais sociocognitifs liés au genre. Nous avons ainsi présélectionné une douzaine de 
pratiques RH appartenant à chacun des trois groupes non exclusifs évoqués plus haut, susceptibles a priori de réduire l'impact des représentations dans la prise de décision.

Dans le groupe "transparence de l'information ", nous avons retenu : l'information des employés sur la formation, la présence d'une campagne d'information sur le droit individuel à la formation (DIF) et un renseignement systématique du nombre d'heures disponibles au titre du DIF.

Le groupe « réflexion structurée autour de la gestion des compétences » est composé des pratiques suivantes : la présence d'une personne ou d'un service responsable de l'organisation de la formation professionnelle continue (FPC), l'existence d'un plan de formation, ainsi que l'évaluation des futurs besoins de qualifications et de compétences.

Le dernier groupe, "dialogue avec les salariés et leurs représentants ", inclut les pratiques suivantes : l'existence d'entretiens professionnels avec l'ensemble du personnel, le fait que l'entretien aborde les questions relatives au développement de carrière et des compétences du salarié et l'implication des représentants du personnel dans la gestion de la formation.

\section{Encadré 2 : La prise en compte des pratiques RH via un indicateur composite}

L'indicateur composite des pratiques RH est construit en suivant une approche de type « faisceau » (Macduffie, 1995), c'est-à-dire en sélectionnant un ensemble de pratiques, fortement corrélées entre elles, dont les effets peuvent se renforcer mutuellement. L'existence d'une pratique présélectionnée sur la base de ses effets " attendus » a été codée par une variable binaire. L'indicateur additif contient 12 items (voir tableau 1), non pondérés $(*)$. L'indicateur est ensuite normalisé pour être compris entre 0 et 1 . Le coefficient alpha de Cronbach vérifiant la cohérence interne de l'indicateur $\mathrm{RH}$ atteint une valeur élevée $(0,87)$, confortant statistiquement notre choix d'items.

Cet indicateur reste une approximation permettant de quantifier les effets d'un ensemble de pratiques sur l'égalité des chances de promotion, sans rendre compte de la complexité du phénomène, ni appréhender la diversité d'usage de chaque pratique.

${ }^{*}{ }^{*}$ : L'absence de pondération permet d'éviter des a priori quant à l'importance d'une pratique par rapport à une autre, tout en soulignant le cumul des items. 
Tableau 1 : Pratiques prises en compte par l'indicateur RH

\begin{tabular}{|c|c|c|}
\hline & Les items de l'indicateur & $\begin{array}{l}\text { Entreprises ayant mis en } \\
\text { place la pratique, } \%\end{array}$ \\
\hline \multirow{3}{*}{$\begin{array}{l}\text { Transparence de } \\
\text { l'information }\end{array}$} & L'employeur informe sur la formation & 79 \\
\hline & Campagne d'information sur le droit individuel à la formation (DIF) & 35 \\
\hline & $\begin{array}{l}\text { L'employeur fournit systématiquement aux salariés un relevé du nombre } \\
\text { d'heures du DIF }\end{array}$ & 58 \\
\hline \multirow{3}{*}{$\begin{array}{l}\text { Réflexion structurée } \\
\text { autour de la gestion des } \\
\text { compétences }\end{array}$} & Existence d'un plan de formation & 47 \\
\hline & $\begin{array}{l}\text { Une personne ou un service spécifique est responsable de l'organisation de } \\
\qquad \text { la FPC en entreprise }\end{array}$ & 37 \\
\hline & L'entreprise évalue ses futurs besoins de qualifications et de compétences & 63 \\
\hline \multirow{6}{*}{$\begin{array}{l}\text { Dialogue avec les } \\
\text { salariés et leurs } \\
\text { représentants }\end{array}$} & Entretiens professionnels avec l'ensemble des personnels & 29 \\
\hline & $\begin{array}{l}\text { Les objectifs individuels de professionnalisation et d'accroissement des } \\
\text { compétences étaient abordés lors des entretiens professionnels * }\end{array}$ & 43 \\
\hline & $\begin{array}{l}\text { Les souhaits des salariés en matière de formation étaient abordés lors des } \\
\text { entretiens professionnels* }\end{array}$ & 47 \\
\hline & $\begin{array}{l}\text { Le déroulement de carrière et l'accès à la formation étaient abordés lors des } \\
\text { entretiens professionnels* }\end{array}$ & 34 \\
\hline & $\begin{array}{l}\text { Les conditions de réalisation d'une formation à l'initiative des salariés } \\
\text { étaient abordées lors des entretiens professionnels* }\end{array}$ & 35 \\
\hline & $\begin{array}{l}\text { Les représentants du personnel sont généralement impliqués dans la } \\
\text { gestion de la formation professionnelle continue }\end{array}$ & 32 \\
\hline
\end{tabular}

Lecture : $29 \%$ des entreprises déclarent pratiquer les entretiens professionnels pour l'ensemble des personnels. Données pondérées du volet Entreprise $(\mathrm{N}=1746)$. Pour les pratiques marquées d'une astérisque $(*)$, la base de calcul des pourcentages représente l'ensemble des entreprises ayant déclaré réaliser les entretiens professionnels.

Source : Difes 2, Céreq, volet Entreprise.

Nous supposons que ces pratiques, fortement corrélées entre elles, peuvent se renforcer mutuellement. Par exemple, lorsque le plan de formation va de pair avec l'implication des représentants du personnel, une analyse des futurs besoins de l'entreprise en compétences et des entretiens systématiques avec les personnels, on peut s'attendre à ce que ces pratiques soient constitutives d'une logique organisationnelle structurée.

Les pratiques RH ne sont pas distribuées uniformément dans les entreprises. Ces pratiques sont globalement plus présentes lorsque l'entreprise est de grande taille (voir tableau A2, en annexe de la version électronique de l'article, et Couprie et Melnik, 2016). Nous introduisons donc les caractéristiques des entreprises dans l'analyse des effets de l'indicateur RH. 


\section{Réduire les inégalités d'accès à la promotion par des pratiques $\mathrm{RH}$}

Après un premier aperçu des écarts constatés entre les hommes et les femmes en termes de taux de promotion et de formation en entreprise, nous analysons l'effet des pratiques $\mathrm{RH}$ sur ces écarts.

\subsection{Promotion et formation en entreprise : des écarts selon le genre?}

Cette partie fournit un premier aperçu des inégalités professionnelles en termes d'accès à la promotion et à la formation, constatées à partir des données Difes2. Les différences observées concernent surtout la promotion.

\subsubsection{Un accès différencié à la promotion selon le genre...}

La promotion en entreprise peut revêtir des formes multiples, selon les organisations, les activités et les métiers (Pergamit et Veum, 1999). Nous définissons la promotion à partir de la question posée aux salariés : "Avez-vous connu un accroissement du niveau hiérarchique dans l'entreprise en 2010 ? ". La promotion correspond ici à une mobilité ascendante dans la hiérarchie de l'entreprise ${ }^{2}$. Ainsi, $15 \%$ des salariés ont connu un accroissement du niveau hiérarchique en 2010 , soit $10 \%$ des femmes et $17 \%$ des hommes. Les taux de promotion moyens varient peu avec la taille de l'entreprise, se situant entre 13 et $16 \%$, sans que ce lien soit croissant.

Tableau 2 : Les taux de promotion par genre et catégorie socio-professionnelle

\begin{tabular}{lcccc}
\hline $\begin{array}{c}\text { Catégorie socio-professionnelle } \\
\text { (CS) }\end{array}$ & $\begin{array}{c}\text { Femmes } \\
\text { promues } \\
\%\end{array}$ & $\begin{array}{c}\text { Hommes } \\
\text { promus } \\
\%\end{array}$ & $\begin{array}{c}\text { Ensemble } \\
\text { promus } \\
\%\end{array}$ & $\begin{array}{c}\text { Part des femmes dans } \\
\text { la CS } \\
\%\end{array}$ \\
\hline Employé administratif & 8 & 12 & 10 & 63 \\
Employé de commerce & 8 & 12 & 9 & 66 \\
Ouvrier qualifié & 14 & 16 & 15 & 10 \\
Ouvrier non qualifié & 8 & 16 & 13 & 42 \\
Cadre & 16 & 20 & 19 & 27 \\
Profession intermédiaire & 10 & 19 & 16 & 31 \\
\hline Ensemble & 10 & 17 & 14 & 37 \\
\hline
\end{tabular}

Lecture : Au sein de la catégorie socio-professionnelle « cadre », où les femmes représentent $27 \%$ des salariés, $16 \%$ des femmes cadres ont obtenu une promotion, contre $20 \%$ des hommes cadres, avec un taux de promotion moyen de $19 \%$.

Source : Difes 2, données pondérées.

2. En cela, notre travail se distingue par exemple de celui de Blasco et al. (2009) quant aux effets de la formation continue sur la promotion (ou la mobilité) sociale, définie comme un phénomène plus large : «un passage à un groupe social de rang supérieur " (12\% de leur échantillon) et/ou le fait que "l'enquêté ait déclaré avoir connu une promotion interne au sein de son entreprise " (8 \% de leur échantillon) (Blasco et al., 2009 p. 39). Au total, $15 \%$ des individus de leur échantillon ont connu une mobilité sociale ascendante. 
Le tableau 2 illustre les taux de promotion des femmes et des hommes selon la catégorie socio-professionnelle (CS). Il révèle la plus forte présence des femmes dans les catégories socio-professionnelles caractérisées par de moindres opportunités de promotion (employés administratifs et de commerce et ouvriers non-qualifiés). Quelle que soit la CS, les écarts de taux de promotion jouent en défaveur des femmes.

\subsection{2... et selon le type de formation suivie}

La promotion et la formation sont deux aspects du développement de carrière reliés et régis par une logique organisationnelle (Macduffie, 1995 ; Solomon et al., 1986). Le lien promotion-formation peut prendre différentes formes. Dans une optique d'accumulation du capital humain, la promotion peut être considérée comme la résultante de la formation en entreprise : les salariés formés valorisent les compétences acquises en formation par le biais d'une promotion (Evertsson, 2004 ; Havet, 2006).

Dans une logique de signalement, de fidélisation ou de reconnaissance, les employeurs proposent les formations aux salariés également susceptibles d'être promus (Beret et Dupray, 1998). Dans les deux cas, les mécanismes de sélection des salariés dans la formation et la promotion jouent un rôle important. Comme le révèlent de nombreux travaux, les salariés qui accèdent à la formation sont aussi ceux qui ont le plus de chances d'être promus. Les travaux quantitatifs prenant en compte ces effets de sélection concluent généralement à la difficulté d'observer un lien causal, tout en soulignant cependant la présence d'un lien fort entre ces deux processus (Blasco et al., 2009).

Tableau 3 : Les hommes et les femmes face à la formation en entreprise

\begin{tabular}{lcc}
\hline & Femmes & Hommes \\
& $\%$ & 38 \\
\hline $\begin{array}{l}\text { Formation « Générale » } \\
\text { (cours, stages, séminaires) }\end{array}$ & 33 & 10 \\
$\begin{array}{l}\text { Formation «Spécifique » } \\
\text { (formation en situation de travail, rotation, autoformation) }\end{array}$ & 11 & \\
\hline
\end{tabular}

Source : Difes 2, Céreq, données pondérées.

Nous supposons en outre que la relation entre la promotion et la formation peut différer selon les types de formation. Certaines formations en entreprise peuvent être plus sélectives que d'autres, selon les objectifs visés ou les coûts plus ou moins élevés. Nous distinguons deux types de formation : "générale " (formations réalisées sous forme de cours, stage ou séminaire) et "spécifique " (formations réalisées principalement en situation de travail) (tableau 3). Notre analyse intègre uniquement les formations financées intégralement par l'entreprise et sur le temps de travail du salarié, très largement majoritaires dans nos données. 


\section{Encadré 3 - La stratégie empirique : formation et promotion, processus reliés influencés par les pratiques $\mathrm{RH}$}

Nous déterminons l'effet des pratiques RH sur la carrière des hommes et des femmes en considérant simultanément leur impact sur la promotion, la formation générale et spécifique. Le modèle probit trivarié autorise l'existence d'un lien quelconque entre les deux types de formation et la promotion, mais aussi entre formation générale et spécifique, sans pour autant induire une relation de causalité. Le modèle prend la forme suivante :

$$
\left\{\begin{array}{l}
P_{i}^{*}=X_{i} \beta_{1}+R_{i} \gamma_{1}+\varepsilon_{i 1} \\
F G_{i}^{*}=X_{i} \beta_{2}+R_{i} \gamma_{2}+\varepsilon_{i 2} \\
F S_{i}^{*}=X_{i} \beta_{3}+R_{i} \gamma_{3}+\varepsilon_{i 3}
\end{array} \quad \text { où } \quad\left(\begin{array}{l}
\varepsilon_{i 1} \\
\varepsilon_{i 2} \\
\varepsilon_{i 3}
\end{array}\right)->N\left(\left(\begin{array}{l}
0 \\
0 \\
0
\end{array}\right),\left(\begin{array}{ccc}
1 & \rho_{12} & \rho_{13} \\
\rho_{12} & 1 & \rho_{23} \\
\rho_{13} & \rho_{23} & 1
\end{array}\right)\right)\right.
$$

Les variables expliquées $P_{i} F G_{i}$ et $F S_{i}$ sont des indicatrices binaires qui représentent respectivement la promotion, les formations générales et spécifiques en 2010 pour l'individu $i$. Les probabilités individuelles sous-jacentes, $P_{i}^{*}, F G_{i}^{*}$ et $F S_{i}^{*}$, sont linéairement reliées à $R_{i}$ l'indicateur de pratiques $\mathrm{RH}$, ainsi qu'à $X_{i}$, matrice des variables explicatives contenant les variables relatives aux caractéristiques de salarié, de son poste et de son entreprise (Tableau A1, figurant en annexe de la version électronique de l'article). Certaines caractéristiques non observées dans nos données (motivations, aptitudes, etc.), peuvent influencer simultanément la sélection des salariés dans la promotion, dans la formation générale et dans la formation spécifique. Ainsi, les termes d'erreur $\varepsilon$ sont reliés par un coefficient de corrélation, $p$, de façon à prendre en compte l'existence de ces facteurs d'hétérogénéité inobservée communs aux trois équations.

Le tableau 4 illustre le lien entre la promotion et les deux types de formation en entreprise. La formation est plus fréquente parmi les promus, notamment chez les femmes. Cependant, la part des non-formés apparaît plus importante pour les femmes non promues : $61 \%$ des femmes non promues n'ont pas été formées, contre $56 \%$ des hommes. Les écarts d'accès à la formation "générale " (cours, séminaires) atteignent 16 points entre les femmes non promues et promues (de 28 à $44 \%$ ), contre seulement 4 points entre les hommes non promus et promus (de 34 à $38 \%$ ). Le même type de constat, avec des écarts moindres, est observé pour l'accès à la formation "spécifique " (en situation de travail). Si les deux types de formation semblent corrélés à la promotion, la formation générale (cours, stages, séminaires) apparaît davantage liée à la promotion que la formation spécifique. 
Tableau 4 : Accès à la formation en entreprise selon la promotion, par genre

\begin{tabular}{lcccc|cccc}
\hline & \multicolumn{3}{c|}{ Promus en 2010, en \% } & \multicolumn{4}{c}{ Non-promus en 2010, en \% } \\
& $\begin{array}{l}\text { FG } \\
\text { seule }\end{array}$ & $\begin{array}{c}\text { FS } \\
\text { seule }\end{array}$ & $\begin{array}{c}\text { FG } \\
\text { et FS }\end{array}$ & $\begin{array}{c}\text { Ni FG, } \\
\text { ni FS }\end{array}$ & $\begin{array}{c}\text { FG } \\
\text { seule }\end{array}$ & $\begin{array}{c}\text { FS } \\
\text { seule }\end{array}$ & $\begin{array}{c}\text { FG } \\
\text { et FS }\end{array}$ & $\begin{array}{c}\text { Ni FG, } \\
\text { ni FS }\end{array}$ \\
\hline Hommes & 38 & 8 & 6 & 48 & 34 & 6 & 3 & 56 \\
Femmes & 44 & 11 & 5 & 40 & 28 & 8 & 3 & 61 \\
\hline Ensemble & 40 & 9 & 6 & 46 & 32 & 7 & 3 & 58 \\
\hline
\end{tabular}

Note : FG : Formation Générale. FS : Formation Spécifique.

Lecture : Parmi les hommes promus en 2010, $48 \%$ n'ont eu aucune formation en entreprise, contre $40 \%$ des femmes promues. En revanche, parmi les hommes non promus en $2010,56 \%$ n'ont pas été formés en entreprise, contre $61 \%$ des femmes non promues.

Source : Difes 2, Céreq, données pondérées.

Nous allons à présent analyser les effets des pratiques $\mathrm{RH}$ sur les chances des femmes et des hommes d'être promus et formés, en reliant les chances de promotion à l'accès à la formation en entreprise. Nous supposons que la relation entre la promotion et la formation peut différer selon les types de formation : plus attachée au poste de travail, la formation spécifique peut être moins sélective et moins reliée à la promotion que la formation générale ayant lieu dans un cadre plus scolaire. L'encadré 3 présente la méthodologie de l'analyse, toutes choses égales par ailleurs, des effets des pratiques RH sur la promotion et les deux types de formation en entreprise considérés simultanément.

\subsection{Les pratiques RH amélioreraient les chances de promotion des femmes en entreprise}

Nous recensons ici les résultats des estimations des effets des pratiques $\mathrm{RH}$ sur les chances de promotion et de formation " générale » (cours, stages, séminaires) et « spécifique » (en situation de travail), toutes choses égales par ailleurs. L'échantillon ainsi que les variables utilisées dans l'analyse sont présentés dans le tableau A1, en annexe de la version électronique de l'article.

Le tableau 5 présente les déterminants des chances d'accès à la promotion et à la formation générale et spécifique selon la méthode décrite dans l'encadré 3 (pour une présentation complète des résultats, voir tableau A4 en annexe électronique de l'article). Des estimations séparées sur les hommes et les femmes sont réalisées car les mécanismes de promotion, de formation et de sélection peuvent différer selon le genre.

Les pratiques RH prises en compte au sein de l'indicateur contribuent à améliorer les chances de promotion des femmes. Les chances de promotion augmentent significativement avec la valeur de l'indicateur $\mathrm{RH}$, et ce pour les femmes uniquement. Les pratiques $\mathrm{RH}$ structurées, favorisant la transparence et le dialogue semblent donc réduire les biais dans les décisions managériales, notamment la sélection dans la promotion. L'analyse 
confirme également qu'une politique RH structurée augmente les chances d'accès à la formation des femmes et des hommes.

Conformément à notre hypothèse, les mécanismes de sélection dans la formation générale et spécifique diffèrent. En effet, les salariés en temps partiel et/ou en contrat à durée déterminée ont significativement moins de chances d'accéder à la formation générale en entreprise que les salariés en CDI (contrat à durée indéterminée) et à temps plein. En revanche, ce phénomène ne s'observe pas dans l'accès à la formation spécifique.

Sans pouvoir affirmer avec certitude le lien causal entre la formation et la promotion, notre analyse confirme l'existence d'un lien entre la formation continue et la promotion. Nos estimations font ressortir un lien de "simultanéité " entre la promotion et la formation. En d'autres termes, les caractéristiques inobservées des salariés ayant de fortes chances d'être formés et celles des salariés qui ont le plus de chances d'être promus sont fortement corrélées, comme cela a déjà été montré dans d'autres travaux (Blasco et al. 2009 ; Havet et Lacroix, 2013).

Le fait de distinguer les deux types de formation en entreprise nous a permis de nuancer le lien formation-promotion selon le genre et selon le type de formation. Pour les femmes, il apparaît que les facteurs inobservés relient la promotion à la formation spécifique (en situation de travail) de la même manière qu’à la formation générale (cours, stages, séminaires). Pour les hommes, un lien significatif entre promotion et formation générale est observé.

Indépendamment du genre, les salariés qui ont plus de chances de bénéficier de la formation générale ont aussi plus de chances d'être promus. Notre hypothèse selon laquelle les logiques de sélection dans la promotion peuvent différer selon le genre et le type de formation semble confirmée. Pour les hommes, la formation spécifique n'apparaît pas significativement liée à la promotion et semble ainsi intervenir plutôt dans une logique de production ou d'adaptation au poste. Pour les femmes, le lien entre formation spécifique et promotion existe, bien qu'il devienne plus faible lorsque l'on utilise une définition alternative de la promotion ${ }^{3}$. Cela laisse supposer que la formation spécifique est mieux valorisée dans les carrières féminines.

Notre conclusion ne rejoint que partiellement celle de Havet et Lacroix (2013) qui soulignaient le rôle bénéfique de la formation sous forme de cours et stages dans les carrières féminines. Ces auteurs montrent, d'une part, que ce type de formation est mieux valorisé en termes de salaire par les femmes et, d'autre part, que le rendement de la formation en situation de travail ou auto-formation n'est pas significatif, sans cependant atteindre celui des hommes.

3. Nous avons conduit une analyse similaire en utilisant une définition alternative de la promotion, vue comme une progression hiérarchique accompagnée d'une hausse de salaire. Notons que l'augmentation de salaire accompagne un peu plus de la moitié des promotions (Couprie et Melnik, 2016). 
Selon les simulations ${ }^{4}$ que nous avons effectuées à partir des estimations obtenues, la valeur maximale de l'indicateur RH ramènerait, toutes choses égales par ailleurs, les taux de promotion féminine au niveau de la moyenne de l'échantillon (14\%). Autrement dit, la présence de l'ensemble des pratiques intégrées dans l'indicateur RH aurait permis une augmentation significative des taux de promotion des femmes.

Tableau 5 : Les déterminants de la promotion et de la formation « générale » et « spécifique»

\begin{tabular}{|c|c|c|c|c|c|c|}
\hline \multirow[b]{2}{*}{ Variables explicatives } & \multicolumn{3}{|c|}{ Femmes } & \multicolumn{3}{|c|}{ Hommes } \\
\hline & Promotion & FG & FS & Promotion & FG & FS \\
\hline Indicateur RH & + & + & + & ns & + & + \\
\hline Temps plein & Réf. & Réf. & Réf. & Réf. & Réf. & Réf. \\
\hline Temps partiel & - & - & ns & - & - & ns \\
\hline CDD & Réf. & Réf. & Réf. & Réf. & Réf. & Réf. \\
\hline CDI & ns & + & ns & ns & + & ns \\
\hline Employé administratif & ns & ns & ns & ns & + & + \\
\hline Employé de commerce & ns & ns & ns & ns & ns & ns \\
\hline Ouvrier qualifié & ns & ns & ns & ns & + & ns \\
\hline Ouvrier non-qualifié & Réf. & Réf. & Réf. & Réf. & Réf. & Réf. \\
\hline Cadre & ns & + & - & ns & + & ns \\
\hline Profession intermédiaire & ns & + & ns & ns & + & ns \\
\hline Rho $_{p-F G}$ & + & & & + & & \\
\hline Rho $_{\text {PFF }}$ & + & & & ns & & \\
\hline $\mathrm{Rho}_{\mathrm{FG}-\mathrm{ES}}$ & ns & & & ns & & \\
\hline Observations & 2015 & & & 3884 & & \\
\hline
\end{tabular}

Note: Ce tableau présente les signes des coefficients obtenus par les estimations probit trivarié robustes à l'hétéroscedasticité. Ns : non significatif au seuil de $5 \%$; + : effet positif et significatif de la variable explicative (en ligne) sur la variable expliquée (en colonne) ; - : effet négatif et significatif. Les variables de contrôle introduites dans le modèle, non présentées ici sont : âge, diplôme, ancienneté, situation familiale, taille de l'entreprise, part des femmes dans les effectifs et secteur d’activité. Les résultats détaillés sont disponibles dans Couprie et Melnik (2016).

$\mathrm{Rho}_{\mathrm{P}-\mathrm{FG}}, \mathrm{Rho}_{\mathrm{P} \text {-FS }}, \mathrm{Rho}_{\mathrm{FG}-\mathrm{FS}}$ sont les coefficients de corrélation des résidus pour les équations de promotion, formation générale et formation spécifique. Lecture : Pour les femmes, l'indicateur RH influence positivement les chances d'accéder aussi bien à la formation qu'à la promotion, tandis que pour les hommes, il ne favorise que les chances d'accéder à la formation.

Source : Difes 2, Céreq.

Le contexte économique et organisationnel de l'entreprise peut impacter l'accès à la formation et à la promotion des salariés. Par exemple, les opportunités de promotion peuvent augmenter ou, au contraire, diminuer avec la restructuration. L'accès à la formation est également relié de manière plurivoque à la conjoncture économique de l'entreprise (Guergoat-Larivière et Perez, 2017). Observe-t-on un lien entre le contexte et l'accès des hommes et des femmes à la formation et à la promotion ? Les effets de contexte domineraient-ils les effets des pratiques $\mathrm{RH}$ ? Pour répondre à cette problématique, nous avons introduit, dans les estimations alternatives, plusieurs variables

4. La méthodologie des simulations et les résultats pour l'accès à la formation générale et spécifique sont détaillés dans Couprie et Melnik (2016). 
relatives au contexte économique et organisationnel : hausse ou baisse d'activité de l'entreprise, une restructuration (variables rapportées par les employeurs), ainsi qu'une variable indiquant une éventuelle introduction d'un nouveau produit, nouvelle technique ou organisation du travail (rapportée par le salarié). Seule cette dernière influence positivement et de manière quasi identique la promotion et la formation des hommes et des femmes, et cela sans affecter les effets de l'indicateur RH. Ce résultat suggère que le changement organisationnel est un facteur potentiellement favorable à la formation et à la promotion, tout en confirmant l'existence d'un effet propre des pratiques RH sur la promotion des femmes. Si le contexte de l'entreprise peut influencer les écarts de promotion et de formation selon le genre, on peut penser que ses effets dépendront en grande partie des pratiques managériales adoptées par l'entreprise.

Enfin, pour affiner notre analyse de l'incidence des pratiques RH sur les inégalités, nous avons réalisé des estimations du modèle en remplaçant l'indicateur synthétique par l'une des douze pratiques qui le constituent, considérées séparément (tableau A3 en Annexe de la version électronique de l'article).

$S$ 'agissant de la promotion des femmes, quatre pratiques ont une influence significative : l'existence d'un plan de formation, la campagne d'information autour du DIF, l'évaluation des besoins en termes de compétences et de qualifications en entreprise, ainsi que le fait d'aborder les souhaits de formation des salariés lors des entretiens professionnels. Quant à la promotion des hommes, aucune pratique n'apparaît positivement reliée aux chances de promotion. L'accès à la formation générale (cours, stages) apparaît globalement plus lié aux pratiques RH faisant partie de l'indicateur que l'accès à la formation spécifique (en situation de travail). Toutefois, comme nous l'avons souligné, une politique de gestion de la formation et de la promotion en entreprise ne se réduit pas à l'existence d'une pratique isolée. L'utilisation d'un indicateur synthétique introduit notamment la possibilité d'effets conjoints liés aux synergies entre des pratiques allant dans le même sens et appartenant à un ensemble cohérent (Macduffie, 1995).

\section{Conclusion}

Nos résultats révèlent l'impact positif et significatif des pratiques structurées de gestion de la formation et des compétences sur les chances de promotion et de formation des femmes. Si les chances de promotion des femmes sont, toutes choses égales par ailleurs, inférieures à celles des hommes, elles augmentent significativement avec la valeur de l'indicateur de la GRH.

Ce travail apporte des éléments quantitatifs quant à la cohérence entre les objectifs de l'égalité professionnelle de genre et une gestion des ressources humaines "fondée sur la transparence et l'objectivité des critères liés aux compétences... " (Laufer et Silvera, 2006, p. 262). Nos résultats confirment également l'idée selon laquelle le recours important 
à la formation, notamment sous forme de cours et stages, va "de pair avec une politique de formation structurée et organisée, une réflexion inscrite dans la durée, une formalisation des pratiques" (Marion-Vernoux, p. 4).

Enfin, l'originalité de notre travail est d'aborder la question des inégalités d'accès à la promotion et à la formation de manière simultanée (comme déjà fait par Havet, 2006 ; Havet et Lacroix, 2013), tout en soulignant le rôle que peuvent jouer certaines pratiques $\mathrm{RH}$ dans la réduction des inégalités.

Un système de pratiques RH structuré, favorisant la transparence et l'objectivité, réduit les biais dans les décisions managériales concernant notamment la promotion. Il ne s'agit cependant pas des pratiques RH visant la formalisation, la standardisation, ni la désindividualisation de la fonction $\mathrm{RH}$ fréquemment citées comme moyens d'objectiver les décisions des managers. Selon nous, une politique RH favorable à l'égalité professionnelle devrait pouvoir allier l'objectivation des décisions à l'existence d'un dialogue à la fois personnalisé et institutionnalisé (Bielby, 2000).

Ainsi, l'accent n'est pas mis ici sur la formalisation ou la bureaucratisation comme moyens de réduire les inégalités professionnelles de genre, mais sur la synergie des différentes pratiques qui créé un cadre procédural suivant une certaine logique organisationnelle. Cette synergie permet d'impliquer les acteurs dans un dialogue autour du développement professionnel des salariés et des besoins en compétences de l'entreprise. En effet, une même pratique peut avoir des effets contraires dans d'autres contextes. Par exemple, on observe certains usages détournés des entretiens individuels annuels qui apparaissent alors non plus comme un outil permettant de prendre en compte les aspirations des salariés, mais comme un instrument de diffusion des "impératifs de rentabilité » de l'entreprise (Benquet, 2013). De même, la représentation du personnel peut ne pas garantir l'égalité professionnelle, faute de parité par exemple (Defalvard et al. 2008).

Notre travail comporte certaines limites, inhérentes notamment à la méthodologie et aux données utilisées. D’une part, nous ne disposons pas de données concernant les démarches volontaristes des entreprises en matière d'inégalités professionnelles. Il nous est donc impossible de croiser les pratiques que certains chercheurs qualifient de "neutres» vis-à-vis du genre (Dencker, 2008) et celles qui visent explicitement à favoriser les femmes. D'autre part, un travail ultérieur pourrait s'intéresser aux différentes configurations des pratiques RH (ainsi qu'à l'organisation de travail par exemple), afin de rendre compte de la diversité des pratiques et de leurs effets.

Notre travail soulève enfin la question de la fonction RH comme mission stratégique de l'entreprise, dans un contexte où les contraintes financières et la logique comptable pèsent sur la stratégie de développement des ressources humaines et des compétences. Le risque est alors que la poursuite des objectifs d'égalité professionnelle, plutôt que de s'inscrire dans une vision stratégique de l'entreprise, ne se limite au simple " contrôle 
de l'application des obligations légales et réglementaires relatives aux conditions et aux relations de travail " ${ }^{5}$. Or, les politiques de gestion des compétences, articulées avec les outils et les politiques de la GRH, et leur appropriation, notamment par les managers intermédiaires, enrichissent les débats et le dialogue social autour de la stratégie de l'entreprise. Elles lui permettent de s'adapter efficacement aux évolutions de son environnement économique et social (Oiry et al. 2013).

Du côté des politiques publiques, actuellement plutôt axées sur l'obligation " de moyens " (obligation de négocier, rapports obligatoires), l'enjeu est de trouver un juste équilibre entre l'injonction et l'incitation, le soutien et la sensibilisation des acteurs (entreprises, mais aussi partenaires sociaux et acteurs du domaine de la formation). Certaines mesures, comme la diffusion des "bonnes pratiques" auprès des entreprises, la création du Label Egalité, vont en partie dans ce sens, mais leur portée reste limitée (Brunet et Dumas, 2012). Les sanctions et l'obligation de négociation collective autour de l'égalité professionnelle sont censées contribuer à inscrire plus fermement la problématique des inégalités de genre dans les préoccupations des acteurs. Cependant, sans une réelle appropriation des enjeux par les acteurs économiques, la portée de ces mesures risque d'être limitée. Dans un tel contexte, les pratiques managériales apparaissent comme des vecteurs potentiels de la promotion de l'égalité professionnelle permettant de mobiliser les forces internes à l'entreprise. Menées en concordance avec l'objectif d'égalité professionnelle, elles pourraient constituer une avancée pour atteindre une transformation plus organique.

\section{Bibliographie}

Becker M., Derrien-Cortés C. (2014), "La négociation collective sur l'égalité professionnelle dans les entreprises de 50 à 300 salariés en 2012 et 2013 ", Rapport du Conseil supérieur de l'égalité professionnelle entre les femmes et les hommes, $\mathrm{n}^{\circ}$ 2014-002.

Belghiti-Mahut S. (2004), « Les déterminants de l'avancement hiérarchique des femmes cadres ", Revue Française de Gestion, n 151, pp. 145-60.

Benquet M. (2013), "Encaisser! Enquête en immersion dans la grande distribution", Paris, La Découverte, coll. « Cahiers libres».

Béret P., Dupray A. (1998), « La formation professionnelle continue : de l'accumulation de compétences à la validation de la performance ", Formation Emploi, n 63 , pp. 61-80.

5. Source : fiche métier en management des ressources humaines, Pôle emploi, direction générale-direction des opérations (Fiche Rome M1503). 
Bielby W. (2000), “Minimizing Workplace Gender and Racial Bias”, Contemporary Sociology, 29(1), pp. 120-129.

Blasco S., Lê, J., Monso O. (2009), "Formation continue en entreprise et promotion sociale : mythe ou réalité ? ", INSEE Références.

Brunet S., Dumas M. (2012), « Bilan de l'application des dispositifs promouvant l'égalité professionnelle entre femmes et hommes ", Etude du Conseil économique, social et environnemental.

Charbeau L., de Larquier G. (2010), «Evaluer une politique d'égalité professionnelle en entreprise : l'apport d'une analyse en termes de parcours ", Formation Emploi, $\mathrm{n}^{\circ} 112$, pp. 5-20.

Cohen P., Huffman M. (2007), "Working for the Woman? Female Managers and the Gender Wage Gap”, American Sociological Review, 72(5), pp. 681-704.

Couppié T., Epiphane D. (2006), «La ségrégation des hommes et des femmes dans les métiers : entre héritage scolaire et construction sur le marché du travail ", Formation Emploi, 93, pp. 11-27.

Couprie H., Melnik E. (2016), «Les pratiques managériales peuvent-elles réduire les inégalités professionnelles de genre ? L'exemple de la promotion et de la formation en entreprise ", Net.Doc. $\mathrm{n}^{\circ}$ 158, www.cereq.fr/content/download/16867/136005/file/ netdoc158.pdf

DARES (2015), "Les écarts de salaires entre femmes et hommes par zones d'emploi ", DARES analyses, mars, $\mathrm{n}^{\circ} 020$.

Defalvard H., Guillemot D., Lurol, M., Polzhuber E. (2008), « Les relations sociales en entreprise : des évolutions à l'épreuve du genre ", in Amossé T., Bloch-London C., Wolff L. (dir.) Les relations sociales en entreprise. Un portrait à partir des enquêtes relations professionnelles et négociations d'entreprise, Paris, La Découverte.

Dencker J.C. (2008), “Corporate restructuring and sex differences in managerial promotion”, American Sociological Review, 79, pp. 455-476.

Dickens L. (1998), "What HRM means for gender equality”, HRM Journal, Vol. 8, $\mathrm{n}^{\circ} 1$, pp. 23-40.

Dobbin F., Schrage D., Kalev A. (2015), "Rage against the Iron Cage: the Varied Effects of Bureaucratic Personnel Reforms on Diversity", American Sociological Review, Vol. 80(5), pp. 1014-1044.

Dubois J.-M., Marion-Vernoux I., Noak E. (2016), "Le dispositif d'enquêtes Defis, un nouveau regard sur la formation en entreprise ", Céreq Bref $n^{\circ} 344$.

Evertsson M. (2004), "Formal On-the-Job Training: A Gender-Typed Experience and Wage-Related Advantage?”, European Sociological Review, Vol. 20, n 1, pp. 79-94. 
Fournier Ch. (2001), «Hommes et femmes salariés face à la formation continue. Des inégalités d'accès qui reflètent les niveaux de qualification et les conditions familiales ", Céreq Bref nº 179.

Gobillon L., Meurs D., Roux S. (2015), "Estimating Gender Differences in Access to Jobs”, Journal of Labor Economics, 33(2), pp. 317-363.

Guergoat-Larivière M. et Perez C. (2017) «Formation continue des salariés en temps de crise : quels liens avec les ajustements pratiqués par leurs entreprises ? ", Formation Emploi 137, janvier-mars.

Guérin F., Pigeyre F., Gilbert P. (2009), "La professionnalisation de la fonction RH. Mythe et limites ", Revue Française de Gestion, 194, pp. 105-121.

Havet N. (2006), "La valorisation salariale et professionnelle de la formation en entreprise diffère-t-elle selon le sexe ? L'exemple canadien ", Economie et Prévision 2006/4, $\mathrm{n}^{\circ} 175-176$, pp. 147-161.

Havet N., Lacroix G. (2013), «La formation continue, un moyen de réduire les inégalités salariales entre hommes et femmes ?", Revue Economique, 64(2), pp. 279-307.

Huffman M.L. (1995), “Organizations, Internal Labor Market Policies, and Gender Inequality in Workplace Supervisory”, Sociological Perspectives, 38(3), pp. 381-397.

Kalev A., Dobbin F., Kelly E. (2006), "Best practices or best guesses? Assessing the efficacy of corporate affirmative action and diversity policies", American sociological review, 71(4), pp. 589-617.

Kalev A. (2009), "Cracking the Glass Cages? Restructuring and Ascriptive Inequality at Work”, American Journal of Sociology, 114(6), pp. 1591-1643.

Klarsfeld A., Ng E., Tatli A. (2012), "Social regulation and diversity management: a comparative study of France, Canada and the UK", European Journal of Industrial Relations, 18(4), pp. 309-327.

Landrieux-Kartochian S. (2007), «Les organisations face au plafond de verre ", Revue Française de Gestion, 173, pp. 15-30.

Larquier (de) G., Marchal E. (2014), “The ways of selecting applicants: diversity speaks in favour of diversity", Document de Travail du CEE, $\mathrm{n}^{\circ} 2014-42$.

Laufer J., Silvera R. (2006), "L'égalité des femmes et des hommes en entreprise : de nouvelles avancées dans la négociation?", Revue de l'OFCE, 97, pp. 245-271.

Macduffie J. (1995), "Human Resource Bundles and Manufacturing Performance: Organizational Logic and Flexible Production Systems in the World Auto Industry", Industrial and Labor Relations Review, 48(2), pp. 197-221. 
Marion-Vernoux I. (2013), "Variété des politiques de formation continue dans les petites entreprises ", BrefCéreq, n 310.

Meurs D., Pailhé A., Ponthieux S. (2011), "Child-related career interruptions and the gender wage gap in France", Economix Working Paper, 2011-03.

Oiry E., Bellini S., Colomer T., Fayolle J., Fleury N., Fredy-Planchot A., Kahmann M., Grimand A., Laval F., Le Guellec T., Lejeune J.-F., Malaquin M., Martin F., Remond A. et Vincent S. (2013) «La GPEC : de la loi aux pratiques RH - identification de quatre idéaux-types ", ESKA, Annales des Mines - Gérer et comprendre 2013/2, n 112, pp. 4-16.

Pergamit M., Veum J. (1999), "What is Promotion?”, Industrial and Labor Relations Review, 52(4), pp. 581-601.

Pochic S. (2005), " Faire carrière : l'apport d'une approche en termes de genre ", Formation Emploi, n 91, pp. 75-93.

Ponchut M., Barth I. (2012), "La place minorée des femmes dans les organisations. Exploration d'un facteur explicatif : la culture organisationnelle. Cas d'une entreprise de transport public ", @GRH 2012/3, n 4, pp. 11-31.

Reskin B., Bielby D. (2005), "A Sociological Perspective on Gender and Career Outcomes", The Journal of Economic Perspectives, 19(1), pp. 71-86.

Ridgeway C., Corell S.J. (2004), "Unpacking the gender system: a theoretical perspective on gender beliefs in social relations”, Gender \& Society, 18(4), pp. 510-31.

Sigot J.-C., Vero J. (2009), « Démocratiser la formation continue dans l'entreprise : le rôle de l'information, des entretiens professionnels et des supports collectifs ", Bref Céreq, n 260.

Solomon E., Bishop R., Bresser R. (1986), “Organizational Moderators of Gender Differences in Career Development : a Facet Classification”, Journal of Vocational Behavior, 29, pp. 27-41. 


\section{$\cdots, 72-1 \cdots \cdots$ \\ REVUE TRIMESTRIELLE \\ RELATIONS INDUSTRIELLES}

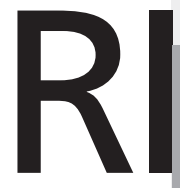

Revue trimestrielle bilingue publiée depuis 1945 par le Département des relations industrielles de I'Université Laval

SYMPOSIUM

Occupational Health and Safety (OHS) /

Santé et sécurité au travail (SST)

GUEST EDITORS/ÉDITEURS INVITÉS : KATHERINE LIPPEL, RICHARD JOHNSTONE AND/ET GENEVIËVE BARIL-GINGRAS

Introduction (anglais/français)

Regulation, Change and the Work Environment Régulation, changement et environnement du travail KATHERINE LIPPEL, RICHARD JOHNSTONE AND/ET GENEVIĖVE BARIL-GINGRAS

ARTICLES

Private Governance, State Regulation and Employment Standards: How Political Factors Shape their Nexus in Australian Horticulture ELSA UNDERHILL AND MALCOLM RIMMER

Protection for Migrant Workers under Evolving Occupational Health and Safety Regimes in China MANKUI LI

Precarious Employment and Difficult Daily Commutes STÉPHANIE PREMJI

«Travailler la nuit pour voir ses enfants, ce n'est pas l'idéal! »

Marge de manœuvre pour concilier vie familiale et horaires atypiques d'agentes et d'agents de nettoyage du secteur des transports

MÉLANIE LEFRANÇOIS, JOHANNE SAINT-CHARLES ET KAREN MESSING

Workplace Death at Sea: Chinese Surviving Families' Experiences of Compensation Claims

DESAI SHAN

Science asservie et invisibilité des cancers professionnels : études de cas dans le secteur minier en France

ANNIE THÉBAUD-MONY

\section{Hors-thème}

Pratiques de gestion des ressources humaines et stratégies d'innovation en France: les apports de l'enquête REPONSE 2011

\section{INDUSTRIAL RELATIONS}

\section{QUARTERLY REVIEW}

A bilingual quarterly published since 1945 by the Industrial Relations Department, Université Laval

\section{RI/IR EN LIGNE}

$\mathrm{RI/IR}$ est disponible en ligne sur le site Érudit :

\section{www.erudit.org/revue/ri}

Pour abonnement institutionnel, contacter Érudit.

Pour consulter les règles de publication ou vous abonner, visitez notre site Internet :

\section{www.riir.ulaval.ca}

\section{RI/IR ONLINE}

$\mathrm{RI} / \mathrm{R}$ is available on line on Érudit website at:

\section{www.erudit.org/revue/ri}

For an institutional subscription to digitalized issues, please contact Érudit.

Visit our website for Notes to contributors or to subscribe:

\section{www.riir.ulaval.ca}

\section{RELATIONS INDUSTRIELLES} INDUSTRIAL RELATIONS

Pavillon J.-A.-DeSève

1025, avenue des Sciences-Humaines Bureau 3129, Université Laval Québec (Québec) Canada G1V 0A6 TÉLÉPHONE : (418) 656-2468 COURRIEL / E-MAIL :

relat.ind@rlt.ulaval.ca

www.riir.ulaval.ca 